- Support for completion of specific audits.

Method A project lead oversaw and drove the project by:

- Developing and delivering training courses and materials;

- Establishing quality improvement projects;

- Supporting staff to complete those projects over a three month period.

Staff were invited to participate by their managers. A series of six workshops covered the stages of audit. The overarching topic was discussed and broken down into different areas for examination by groups. The groups were supported with their individual projects.

Results Six projects included communication, information sharing and timings. Participants agreed items of most concern/ interest, learned where to find evidence and standards; how to design tools, collect, analyse and present data. Participants enthusiastically presented results and identified areas for improvement together. Groups were multidisciplinary and demonstrated benefit from examining processes objectively.

Discussion/conclusions Support through tailored workshops and individual advice meant each group completed their project and participants were enthusiastic in championing changes to improve care. Confidence increased and multi-professional groups worked well together. Further refinement could improve the programme which is to be repeated with a different focus. Lessons learnt included ensuring good communication about the programme and getting the timings of workshops right.

\section{P-154 IMPROVING QUALITY IN A HOSPICE}

Anne Bisset-Smith, Jane Naismith. St. Joseph's Hospice, London, UK

\subsection{6/bmjspcare-2019-HUKNC.176}

Background Previously, the hospice instituted changes in the light of national guidance and themes from incidents, but the manner in which these changes were implemented lacked quantitative proof that the change was an improvement. Also, insufficient attention was paid to defining the root cause of an issue and the possibility of unintended negative consequences.

Aim For any clinical improvements to be pursued using the Institute for Healthcare Improvement (IHI) model of quality improvement (QI). This includes clearly defining the aim, using a baseline measure to clarify the issue, putting a specific intervention into place and using outcome and balancing measures to check whether the change constituted an improvement.

Method A 'Quality Improvement and Clinical Governance Lead' post was created and filled in November 2018.

The post holder:

- Delivers QI teaching on a monthly basis, with the aim of $60 \%$ of clinical staff being trained by March 2020;

- Attends all clinical incident review meetings and assists the team to address themes using QI methodology;

- Oversees all patient feedback in the same manner;

- Supports individuals and teams to run QI projects.

Results By June 2019, 17 members of staff had been trained. The initial session was delivered to multi-professional managers and then rolled out to all clinical staff. The feedback from the sessions was positive;

'Step by step process to support QI'.

'Will enable me to measure QI improvement'.

'Helped to organise some of my own ideas and study my own PDSA cycle'.

This training, together with the presence of the QI lead in all patient quality meetings, has led to proposed changes across the hospice being designed and evaluated using the QI model.

Conclusions The use of QI methodology has led to more sustainable and effective interventions and has given the hospice clearer evidence of our improvement journey.

\section{P-155 EFFECTS OF A WEEKLY HOSPICE BASED EXERCISE GROUP ON TIMED UP AND GO (TUG) AND PATIENT SATISFACTION}

Miriam Lemon. Wirral Hospice St John's, Higher Bebington, UK

\subsection{6/bmjspcare-2019-HUKNC.177}

Background Patients with life-limiting illnesses often experience functional impairment, resulting in poorer quality of life (QOL). Exercise can improve physical, emotional and psychological wellbeing which help maintain independence and improve QOL (Wittry, Lan \& McNalley, 2018). A successful chair-based exercise group is well established, however, a need was identified for a more challenging group for patients with a higher level of functional mobility.

Aims of the project To establish an exercise group to provide a relaxed and sociable environment to promote strength and balance exercises. To investigate changes in mobility after attending a weekly exercise group. To investigate patient satisfaction for the service. To empower patients to self-manage their condition and continue with exercise at home.

Methods Patients were assessed using the TUG outcome measure (Podsiadlo \& Richardson, 1991) on initial assessment. Patients attended a weekly exercise group under the supervision of a physiotherapist with volunteer support, involving exercises to improve strength and balance. TUG was repeated at intervals of four attendances and a satisfaction questionnaire was completed at the end of eight sessions.

Results All attendees showed improvements in TUG with mean reduction in walking time of 3.64 seconds (range: 0.25-8.46 seconds). After four exercise sessions 71\% of patients had a TUG score of 14 seconds or less - suggesting a lower risk of falls. Satisfaction with the group was high with many positive themes being reiterated including selfesteem, self-confidence and motivation; and no negative feedback received. $100 \%$ of responders enjoyed the exercise group and found it relevant to them. 100\% agreed or strongly agreed that the exercise was at an appropriate level and alternative exercises were suggested when necessary for individual ability.

Conclusion The exercise group has proved to be a positive addition to hospice services and demonstrated that simple strength and balance exercises are effective in improving both physical and psychological health in this patient group. 